\title{
Neue Wege in der Ausbildung von Verfahrenstechnikern - Erfahrungen im Modul Anlagenbau mit Projektarbeiten
}

\author{
Karlheinz Kuchling, Eberhard Wegener
}

Projektarbeiten mit dem Ziel komplexe Aufgabenstellungen zu bearbeiten, waren von Anbeginn Bestandteil des Studienfaches Anlagenbau. Bei einer gemeinsamen Aufgabenstellung hatte jedoch jede/r Studierende aufgrund unterschiedlichen Durchsatzes auch eine unterschiedliche Lösungsvariante für die Anlagengröße und -gestaltung. Neu hingegen war der Versuch, diese Aufgabenstellung im Rahmen des Projektmanagements unter Verantwortung (mit Unterstiitzung und Anleitung) eines studentischen Projektleiters durch verschiedene Arbeitsgruppen - entsprechend den Fachgewerken in der Praxis - in einem höheren Detailliertheitsgrad abzuarbeiten. Die vor- und nachteiligen Ergebnisse werden vorgestellt.

\section{Ausgangssituation}

Bei der Entwicklung des Curriculums für den Studiengang Verfahrenstechnik stand die Einheit von Verfahren - Apparat - Anlage im Vordergrund. Dies war einerseits den beruflichen Erfahrungen der berufenen Hochschullehrer entsprechend, andererseits den gewandelten Anforderungen der Praxis geschuldet, die sich nur langsam im Ausbildungsrahmen von Verfahrensingenieuren niederschlugen. Die Diskussion hierzu wurde von KLAPP [1] eingeleitet und fand ihre Fortsetzung $u$. a. durch SCHWIND [2], GELBE [3], SCHLACHTER [4] sowie FREY [5]. Sie endete letztlich in dem Memorandum „Verfahrenstechnik und Chemieingenieurwesen im Wandel " [6] und dem Rahmenstudienplan Verfahrenstechnik der GVC [7]. Verbunden mit dem Beitritt der TFH Wildau zum Fachbereichstag Verfahrenstechnik 1992 und der hierzu notwendigen Anerkennung des o. a. Rahmenstudien- planes wurde die Grundlage für die apparative und anlagentechnische Ausbildung als wesentlicher Ausbildungsinhalt von Verfahrenstechnikern gelegt. Im Verlaufe der Jahre erfolgte eine kontinuierliche Weiterentwicklung des Studienfaches Anlagenbau, insbesondere die Erhöhung der Anforderungen bei den praxisnahen Belegthemen, die in starkem Maße die Anwendung erworbenen Wissens aus anderen Studienfächern erforderte.

Neben der einschlägigen Fachliteratur steht den Studierenden ein Vorlesungsskript zur Verfügung. Es beinhaltet neben der Beschreibung der aus der Verfahrensentwicklung resultierenden Dokumentationen wie Verfahrensgrundfließbild, Verfahrensfließbild und $R+1$ Schema (Rohrleitungs- und Instrumentierungsschema) die für das Basic- und Detail-Engineering notwendigen Angaben zur Standortfestlegung und Lageplangestaltung, zur Trassen- und Baufeldgestaltung, Aufstellungsplanung, zu Fundament- und Durchbruchplänen, zur Rohrleitungsplanung und Erstellung von Isometrien als Arbeitsgrundlage für die Projektbearbeitung.

Für das Studienfach wurde bisher etwa $1 / 4$ der zur Verfügung stehenden Zeit für die theoretischen Grundlagen verwendet, $1 / 2$ für die Projektbearbeitung einschließlich der notwendigen Konsultationen und Testate und $1 / 4$ für die CAD-Ausbildung. Zu Beginn der Ausbildung wurde nach einem Werkzeug gesucht, welches eine durchgängige Planung ermöglicht. Sicher war diese Forderung sehr weit gefasst mit einem System über den gesamten Bereich und auch noch kostengünstig für die Lehre. Nur so ist aber annähernd das Stoff/Zeit/Geld-Problem zu lösen. Mehrere Systeme wurden untersucht. In die Endauswahl kamen zwei Softwarepakete. Aus Kostengrün-

\section{Materialwirtschaft}

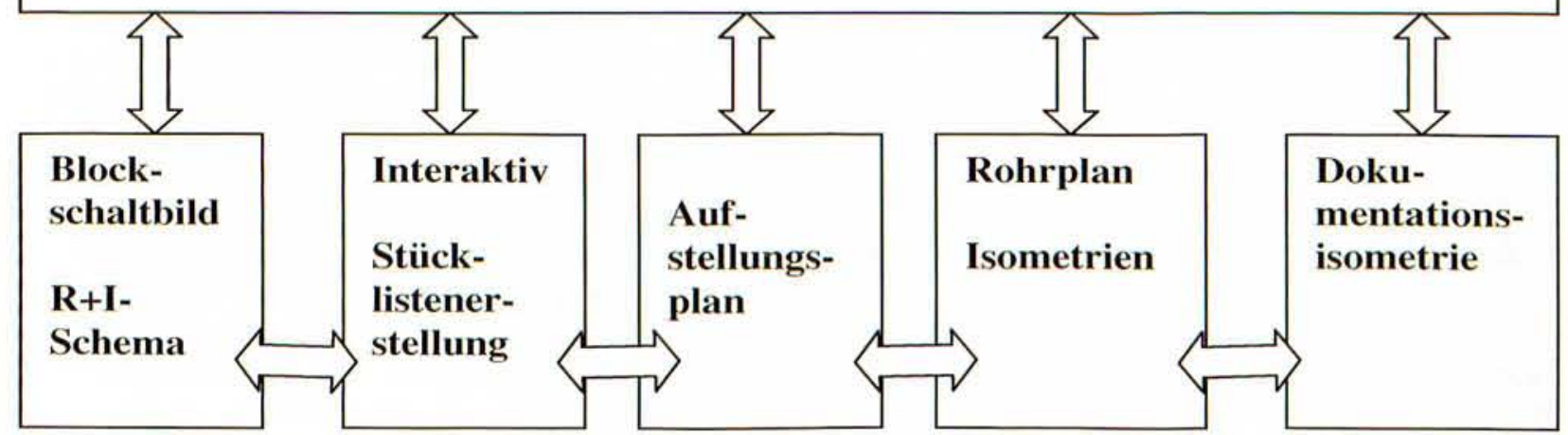

Abb. 1: Modularer Aufbau CADdy-Anlagenplanung 
den wurde eine Entscheidung für das System CADdy getroffen. Dieses System der Firma Ziegler Informatics $\mathrm{GmbH}$ in Mönchengladbach ist modular aufgebaut (Abb. 1).

Nicht nur die Anlagenplanung ist modular aufgebaut, sondern universelle CADdy-Bausteine erleichtern die Arbeit zusätzlich. Dazu kann man beispielsweise die Programmierschnittstelle zählen. Die systemeigene Programmierschnittstelle CADdy-PLUS basiert auf der Sprache C. Damit werden Möglichkeiten eröffnet, die weit über eine Variantenprogrammierung hinausgehen (Böden in unterschiedlichsten Varianten).

Selbst bei größeren Projekten behält ein Anwender mit der Zeichnungsverwaltung eine ausgezeichnete Übersicht. Zeichnungen, Pläne, Dokumentationen werden auf Datenbankbasis (dBASE-Format) verwaltet. Damit ergab sich eine elegante Verbindung zu Excel. Durch diese Verbindung konnten auch Angebote effektiv ausgearbeitet werden. Zum Datenaustausch mit anderen Systemen stehen standardisierte Schnittstellen wie DXF, DWG, IGES und ASCIl zur Verfuigung.

Die Beschaffung weiterer Software für die Rohrleitungsplanung wurde aufgrund für die Anwendung in nur einem Studienfach und auch wegen der geringen Studentenzahlen ausgeschlossen, zumal über CADdy der Einstieg in anspruchsvolle Software wie Intergraph möglich ist, wie die Anlagenplanung von BECKER [8] bewiesen hat.

Probleme bei der bisherigen Belegbearbeitung traten insbesondere dort auf, wo Selbtständigkeit und Übertragungsfähigkeit von Grundregeln gefordert waren, z. B. bei der Aufstellungsplanung der Ausrüstungen und der Rohrleitungsplanung. Hier machten sich fehlendes Vorstellungsvermögen und Mängel im Lesen und Verstehen zeichnerischer Unterlagen besonders bemerkbar (dies fuihrte z. B. im modularen System zur Einfuihrung eines Moduls „Konstruktionsgrundlagen/CAD III“ für Verfahrenstechniker), obwohl den Studierenden umfangreiches Bildmaterial sowie zeichnerische Dokumentationen aus dem praktischen Anlagenbau zur Verfügung standen. Es kam dann auch zu einem deutlichen Motivationsabfall bei der Belegarbeit. Es bot sich daher an, die - möglicherweise zu anspruchsvoll angelegte - Aufgabenstellung in einer anderen Form durchzuführen, ohne das Anforderungsniveau zu senken. Bestärkend hierfür ist auch die Forderung der Unternehmen, wie sie auf der ACHEMA 2000 und der GVC-Jahrestagung vorgetragen wurden. Danach sind die Grundlagen der Anlagenplanung (bis zur Inbetriebnahme) in der Ausbildung von Verfahrenstechnikern unabdingbar, unabhängig davon, ob die Absolventen später die Aufgaben des Auftraggebers oder eines Auftragnehmers wahrnehmen werden.

\section{Aufgaben- und Zielstellung des Projektes}

Die gestellte Aufgabe beinhaltet die kontinuierliche Erzeugung einer vorgegebenen Menge/Zeiteinheit von Warmwasser als Wärmeträger für eine Prozessanlage. Dabei tritt als erschwerend auf, dass ein Teilstrom dis- kontinuierlich entnommen wird, zusätzlich ein Verlust innerhalb der Anlage auftritt und die Restmenge im Kreislauf unter Ersatz der Verluste gefahren wird. Als Basisenergie stehen Elektroenergie, Hochdruckdampf und Hochdruckkondensat zur Verfügung. Die räumliche Einordnung der zu entwickelnden Anlage ist als Randbedingung vorgegeben. Die Anlage ist mit ihren Bestandteilen zu entwerfen. Für die Realisierung und den Betrieb sind die erforderlichen verbalen und zeichnerischen Unterlagen zu schaffen. Nach den üblichen Methoden, wie auch von STEINBRUCH/SPITTER [7] beschrieben, sollten eine Organisationsanalyse durchgeführt und Mängel möglichst vermieden werden.

\section{Durchführung des Projektes}

Nach einer eingehenden Erläuterung der Aufgabenstellung wurden nach dem Prinzip der Dekomposition Einzelaufgaben abgeleitet und hierfür studentische Arbeitsgruppen gebildet. Sie sollten sich in die Aufgabe des auftragnehmenden Fachbetriebes versetzen und unter Nutzung der Kommunikationsmöglichkeiten mit den Fachkollegen im Hause als auch mit den auf diesen Gebieten auf dem Markt vertretenen Unternehmen möglichst eigenständig die Projektteile erarbeiten. Für die Koordination und Federführung wurde ein Student als Projektleiter eingesetzt, der betreuende Hochschullehrer vertrat in diesem Rollenspiel den Auftraggeber.

Zur Sicherung des Abgabetermines wurden 3 Testate als Meilensteine festgelegt, um die Arbeitsfähigkeit der Arbeitsgruppen zu gewährleisten. Alle Konsultationen, notwendige Festlegungen und Einschränkungen wurden grundsätzlich im Beisein des Projektleiters durchgeführt bzw. von diesem veranlasst. Die Arbeitsteilung ermöglichte eine intesivere Auseinandersetzung mit den Teilthemen und damit auch eine Erhöhung des Aussageniveaus. Damit das Gesamtverständnis für die Anlagenentwicklung entwickelt und erhalten bleibt und eine gemeinsame Ausgangsbasis geschaffen wird, wurde die Aufgabenstellung bis zum $\mathrm{R}+\mathrm{I}$-Schema gemeinsam seminaristisch erarbeitet:

- Ermittlung der erforderlichen Wärmemenge, des wirtschaftlichen Energieeinsatzes (Elektroenergie, Hochdruckdampf, Hochdruckkondensat, Niederdruckdampf aus Hochdruckdampf oder Entspannung von Hochdruckkondensat),

- darauf aufbauende Erarbeitung des Verfahrens-Grundfließbildes,

- Auslegung der Hauptausrüstungen in Größe und Anzahl (Rohrbïndel-Wärmeübertrager, Entspanner, Druckminderstation, Kondensatrückspeiseanlage, Misch- und Brauchwasserbehälter),

- darauf aufbauende Ausarbeitung des Verfahrensfließbildes,

- Verknüpfung der Ausrïstungen (Kopplungen) durch Rohrleitungen, notwendige Bypässe, Armaturen, Sicherheitseinrichtungen und MSR-Technik in Form eines Entwurfes für das R+I-Schema, welches dann von den Arbeitsgruppen entsprechend zu ergänzen bzw. zu verändern war. 


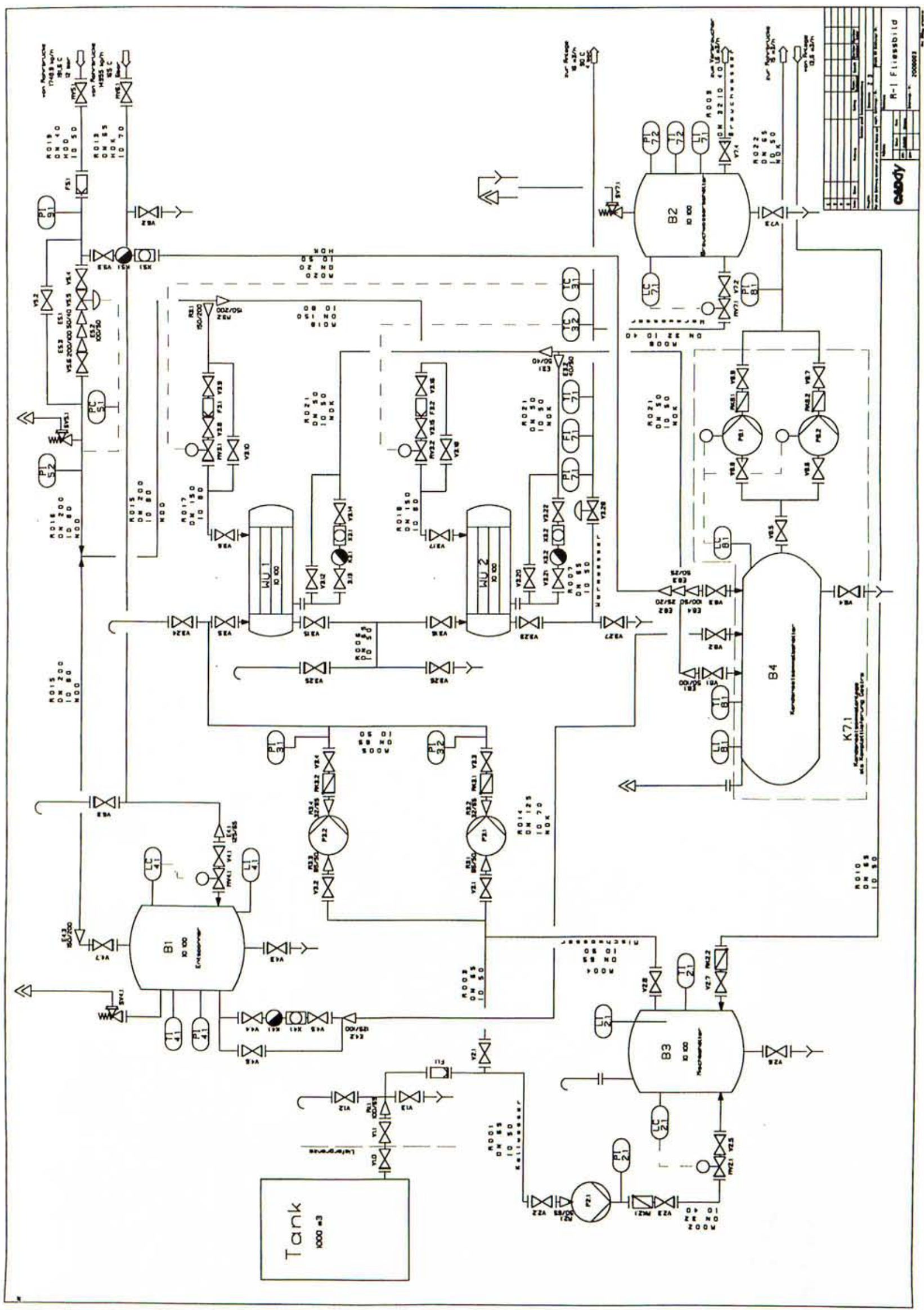

Abb. 2: $R+1$-Fließbild der Anlage 


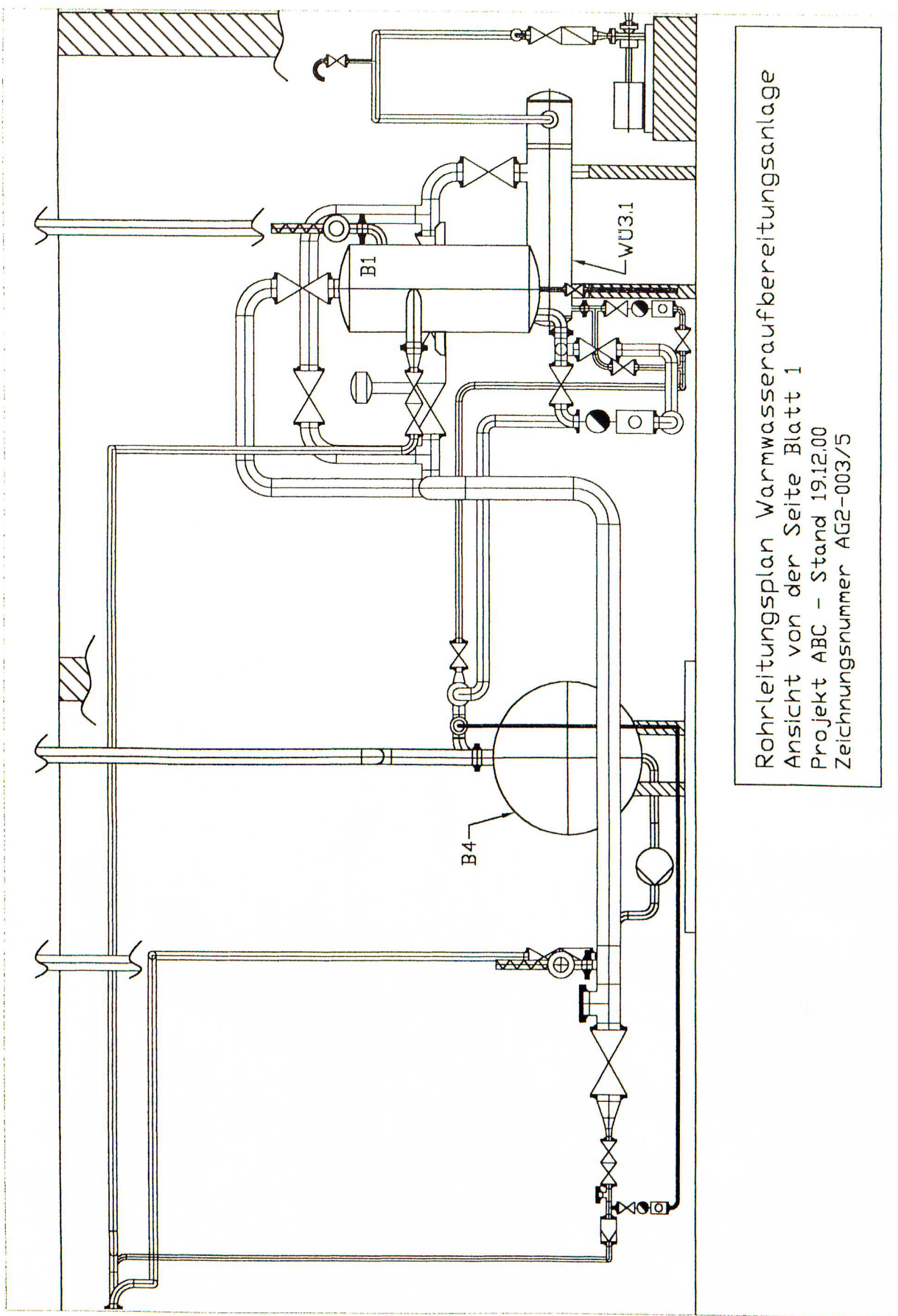

Abb. 3: Teil-Rohrleitungsplan der Anlage 


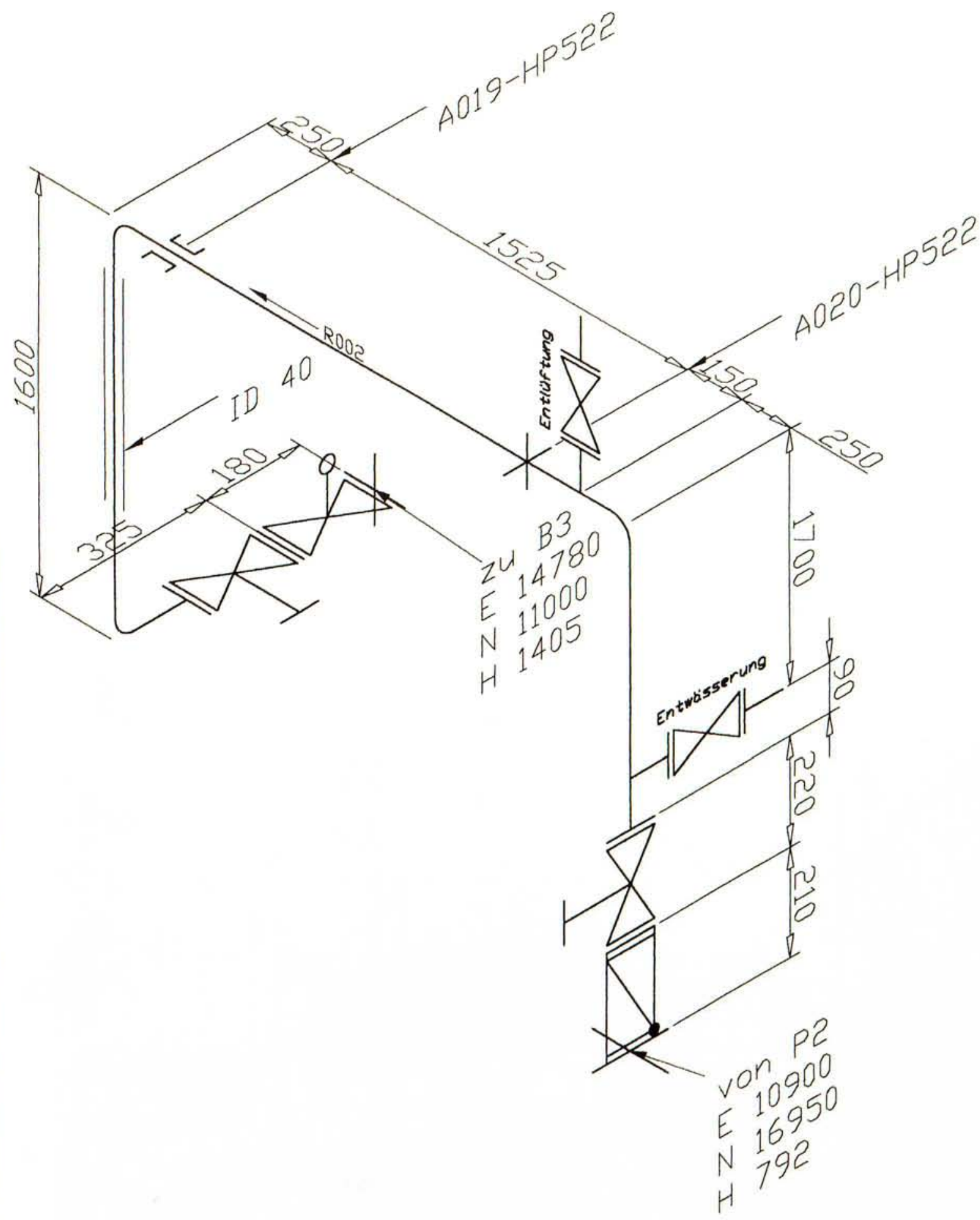

Alle Rohrbögen $3 \times \mathrm{Da}$

$E$ - Dst

$N$ - Nord (bezüglich Koordinaten-0-Punkt)

$\mathrm{H}$ - Hòne über Hallenboden

Rohrleitungsisometrie R002 Projekt ABC - Stand 19.12.00

Zelchnungsnummer AG002-004/2

Abb. 4: Rohrleitungs-Isometrie 


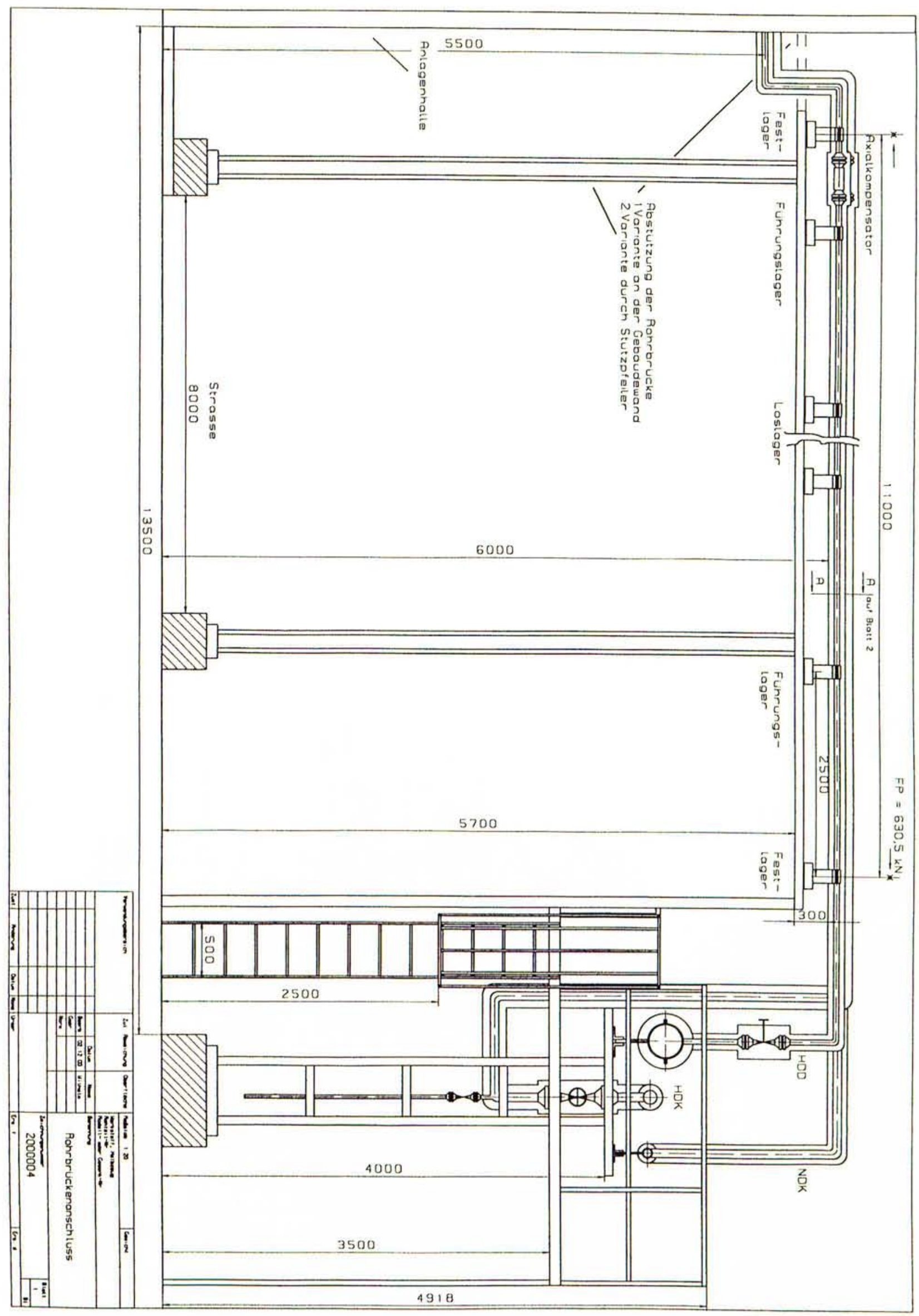

Abb. 5: Aufgabenstellung Bau - Rohrbrücke 
Darauf aufbauend hatten sich die Arbeitsgruppen damit zu beschäftigen, wer wann Informationen von wem erhalten muß oder selbst entsprechende zu liefern hat, um die Aufgabe lösen zu können, und Forderungen abzuleiten. Dies stellte offensichtlich ein neues Problemfeld dar; da es höhere Anforderungen in ungewohnter Weise an die Eigenverantwortlichkeit der Themenbearbeiter stellte, zumal die notwendige Übereinstimmung an den Schnittstellen auch zu unliebsamen Änderungen bereits vorliegender Unterlagen führte.

Den Arbeitsgruppen wurden folgende Aufgaben übertragen:

\section{AG 1:}

Auswahl und Festlegung aller Rohrleitungen (einschl. Entliiftungs- und Entleerungsmöglichkeiten), Werkstoff, Nennweite und Nenndruck, Armaturenauswahl für alle Medien, Druckverlustberechnung, Pumpenauswahl sowie Bestimmung der Dämmungsdicken für die Vermeidung von Wärmeverlusten und die Bildung von Schwitzwasser sowie als Berührungsschutz.

\section{AG 2:}

Aufstellungsplanung für die Ausrüstungen anhand der vorgegebenen baulichen Situation unter dem Gesichtspunkt der Distanzminimierung der Rohrleitungen, der Montage sowie der Anforderungen aus Betrieb, Wartung und Instandhaltung. Dazu Rohrleitungspläne einschließlich Stützweiten, Wärmedehnung, Rohrleitungshalterungen und Rohrleitungsisometrien.

\section{AG 3:}

Ausarbeitung der Aufgabenstellung für das Baugewerk und die zu schaffende Montagefreiheit. Fundamentpläne für die Ausrüstungen, Durchbruchpläne für die Rohrleitungen, technologischer Stahlbau (Rohrleitungsbrüicke, Bühnen und Steigeleitern).

\section{AG 4:}

Auswahl der Gerätetechnik und Festlegung der Einbaubedingungen für die durchsatzabhängige Temperaturregelung der Wärmeiibertrager, Druck- und Temperaturanzeigen an den Rohrleitungen und Apparaten, Füllstandsanzeige und -regelung an den Apparaten, Havarieschaltung an der Rohrbrïcke, elektrische Begleitheizung für Freileitungen, Ausarbeitung der Aufgabenstellung für die E-Technik.

\section{AG 5:}

Montageablaufplanung einschließlich der notwendigen Druck- und Dichtheitsprüfungen, Dämm- und Anstricharbeiten. Technologischer Erläuterungsbericht für die Anlage. Beschreibung der Inbetriebnahme der Anlage, Betrieb und Abfahren, Hinweise für Wartung und Instandhaltung.

\section{AG 6:}

Konstruktionsunterlagen und Statik für den Misch- und den Brauchwasserbehälter in stehender Ausführung gemäß der verfahrenstechnischen Auslegung.
Dem Projektleiter wurden die Koordinierung der Arbeiten, Terminkontrollen, Problemklärungen mit dem Auftraggeber sowie die Zusammenfassung der Ergebnisse der Arbeitsgruppen in Form und Inhalt in Form einer Projektdokumentation ïbertragen. Als ein Teilergebnis sind das durch die Arbeitsgruppen überarbeitete $\mathrm{R}+\mathrm{I}$ Schema der Anlage (Abb. 2), ein Auszug aus der Aufstellungs- und Rohrleitungsplanung (Abb. 3), eine ausgewählte Rohrleitungs-Isometrie (Abb. 4) und die Aufgabenstellung Bau für die zu errichtende Rohrbrüicke (Abb. 5) vorgestellt.

Zur Durchführung des Projektes musste auch erfolgreich ein Datenmanagement geführt werden. Dabei wurden Intranet/Internet-Technologien eingesetzt. Sämtliche Daten wurden einem entsprechenden Archivierungsund Sicherungskonzept anvertraut.

\section{Ergebniseinschätzung}

Diese erstmalige Form der Projektabwicklung wird aus der Sicht des Lehrenden vom inhaltlichen Ergebnis her als sehr gut eingeschätzt, da sie ein qualitativ höherwertiges Ergebnis brachte als die bisherige Projektbearbeitung. Im Kernpunkt der Aufgabenstellung stand die Methodenlehre. Als förderlich für die Entwicklung des Teamgeistes und die Eigenverantwortlichkeit der eigenen Arbeitsleistung zeigte sich die notwendige inhaltliche und zeitliche Abstimmung der Arbeitsgruppen untereinander und die Möglichkeit, mit Fachunternehmen direkt für die Aufgabenstellung in Verbindung zu treten, was von einigen Studenten in anerkennenswerter Weise in Anspruch genommen wurde. Unguinstig wirkte sich die Arbeitsteilung dadurch aus, dass sich nicht jede Arbeitsgruppe unbedingt mit den Ergebnissen der anderen beschäftigen musste, um zum eigenen Ziel zu kommen, d. h. die Gesamtkenntnis hängt sehr vom eigenen Interesse des Bearbeiters ab. Um diesen Sachverhalt zu verbessern, wurde eine umfangreiche Präsentation der Planungsunterlagen vorgenommen, bei der der Projektleiter die Gesamtaufgabenstellung und ihre Erfuillung darlegte und alle Arbeitsgruppen ihre Problemstellungen und Lösungen vorgetragen haben.

Wie bei jeder Teamarbeit zeigte sich die Zusammensetzung des Teams entsprechend Abbildung 6. Dem studentischen Projektleiter bereitete dies schon aufgrund seiner Stellung als „primus inter pares“ erhebliche Probleme. Doch er bemühte sich, diese selbstständig zu lösen.

Die Bewertung aller Projektteilnehmer erfolgte studienbegleitend durch die Testate, die Qualität der jeweils ausgearbeiteten Dokumentation und den Vortrag.

Inwieweit die Einschätzung mit der der Studierenden uibereinstimmt und ob aus ihrer Sicht noch ganz andere Gesichtspunkte eine Rolle spielen, wird in einem gesonderten Beitrag vom Projektleiter der Seminargruppe V 97, Herrn Fahrig, dargelegt. 


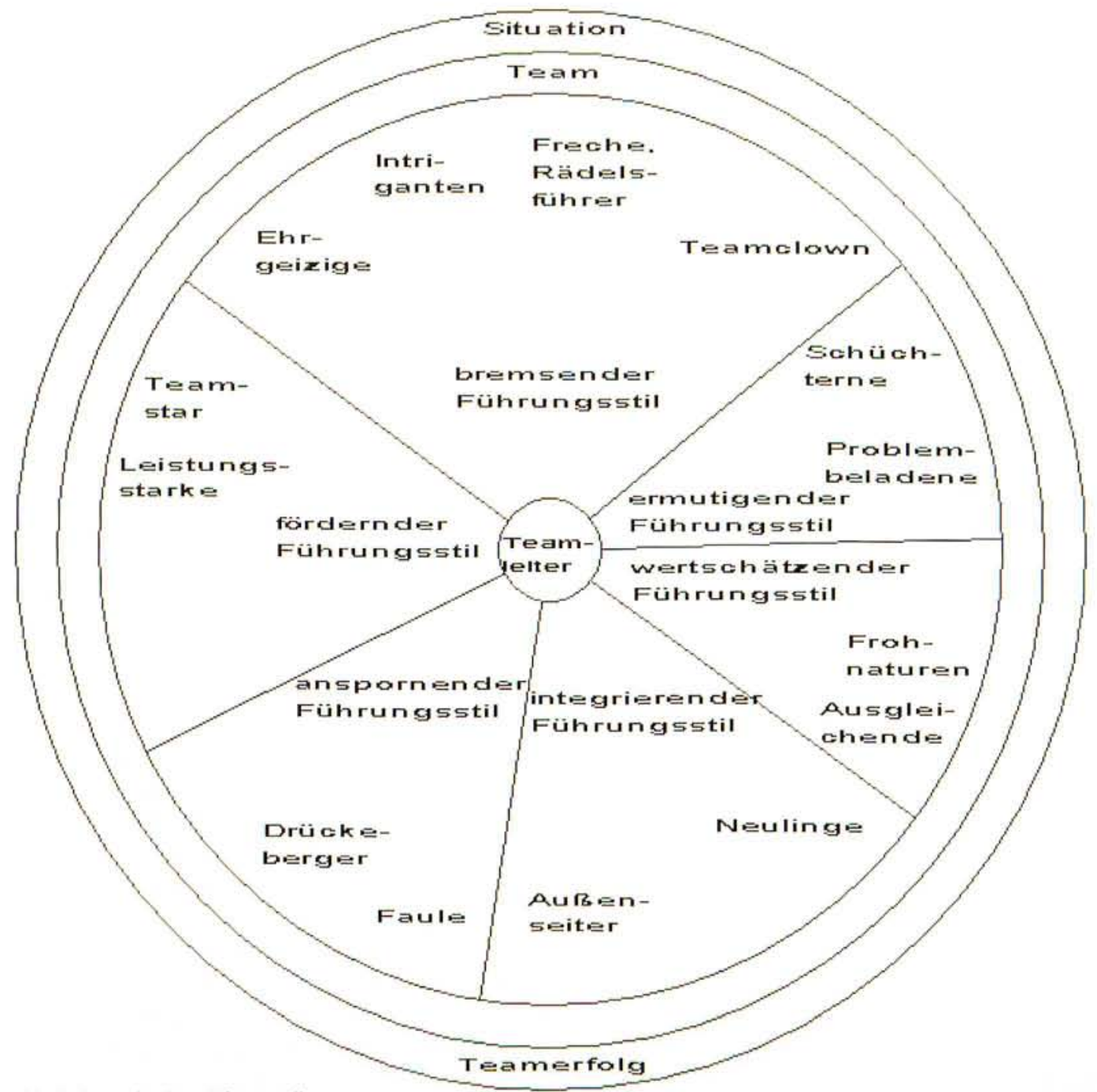

Abb. 6: Teamorientierte Führungsstile

\section{Literatur}

[1] Klapp, E.: Apparatebau/Anlagenbau als Lehr- und Forschungsziel an einer wissenschaftlichen Hochschule. verfahrenstechnik 6(1972)12, S. 385-389

[2] Schwind, H.: Wechselbeziehungen zwischen Anlagentechnik und Apparatekonstruktion, Chem. - Ing. - Techn. 46(1974)13, S. 551-554

[3] Gelbe, H.: Die Apparate- und Anlagentechnik in der Ausbildung von Verfahrens- und Apparateingenieuren, Chem. - Ing. - Techn. 49(1977)7, S. 546-548

[4] Schlachter, H.: Der Verfahrensingenieur in der chemischen Industrie, Chem. - Ing. - Techn. 50(1978)10, S. 775-782

[5] Frey, W.: Wandel in der Verfahrenstechnik - Anforderungen an die Ausbildung, Chem. - Ing. - Techn. 67(1995)2, S. $155-159$

[6] Memorandum: Verfahrenstechnik und Chemieingenieurwesen im Wandel. Empfehlungen für eine zukunftsorientierte Ausbildung und Forschung im Fachgebiet Verfahrenstechnik und Chemieingenieurwesen, Chem. - Ing. Techn. 69(1997)8, S.1014-1016

[7] Steinbruch, Pitter: Betriebliche Informatik, Fr. Kiehl Verlag Ludwigshafen, 1991

[8] Haasis: CIM - Einführung in die rechnerintegrierte Produktion, Hanser-Verlag München, Wien, 1993

\section{Autoren}

Prof. Dipl.-Ing. Karlheinz Kuchling

Technische Fachhochschule Wildau

Fachbereich Ingenieurwesen/Wirtschaftsingenieurwesen Tel. (0 33 75) 508-124

E-Mail: kuchling@wi-bw.tfh-wildau.de

Prof. Dr.-Ing. Eberhard Wegener

Technische Fachhochschule Wildau

Fachbereich Ingenieurwesen/Wirtschaftsingenieurwesen Tel. (0 33 75) 508-169 